\title{
Conformational Analysis of Starch Derivatives by FTIR Spectroscopy
}

\author{
Chris Bruijnes, Ron Bosman, Peter Bareman and Arie Besemer \\ Netherlands Organization for Applied Scientific Research, TNO, \\ Division of Technology for Society, Department of Analytical Chemistry and of Biotechnology \\ P.O. Box 217, 2600 AE DELFT, The Netherlands
}

\begin{abstract}
SUMMARY
Infrared spectroscopy appears to be a helpful tool for conformational analyses of starch derivatives. In this study spectral changes in the fingerprint region between 1200 and $900 \mathrm{~cm}^{-1}$ are related to changes in tertiary structures of $\beta$-cyclodextrin and linear dextrin inclusion complexes, linear dextrin after removal of complexed agent and amorphous amylose. The assumed similarity between the structures of $\beta$-cyclodextrin and linear dextrin inclusion complexes is confirmed by the spectra.
\end{abstract}

\section{INTRODUCTION}

Starch mainly consists of two polysaccharides, amylopectin (70-80\%) and amylose (20-30\%). Amylopectin is a highly branched polymer based on 1-4 and 1-6 linked $\alpha$-D-glucopyranosyl units. The amylose fraction consists of linear chains of 1-4 linked $\alpha$-D-glucopyranosyl units only. The degree of polymerization (Dp) can reach 1000 to 10,000 . Starch occurs naturally in different confirmations in wheat, maize etc. It is generally accepted that amylopectine is the most crystalline fraction in the starch granule. When heated starch solutions are cooled down a more disordered fraction is precipitated, consisting mainly of amylose. This solubility phenomenon is called retrogradation. Traditionally, the process of retrogradation of starch has been examined mainly by X-ray diffraction, but recent studies reported the use of Fourier Transform Infrared Spectroscopy in this field (1). Although the most informative part of the infrared spectra of starch lies between 1,200 and $900 \mathrm{~cm}^{-1}$ very little is known about the assignments of absorption bands in this fingerprint region.

This study reports the results of FTIR measurements on specific starch derivatives, the conformations of which are more or less known, viz. $\beta$-cyclodextrin ( $\beta-C D)$ and linear dextrin inclusion complexes (LD). The invention of production of these linear complexes with $n$-butanol is claimed by the Hayashibara patent (2). The LD complexes are described as crystalline, but no structural information based on analysis is given. The two dextrin types have similar complexing properties with e.g. $n$-butanol. The tertiary structure of the $\beta-C D$ is described as a truncated cone with the glucose units in the C-1 form. The C-2 and C-3 hydroxyl groups of the adjacent glucopyranose units form hydrogen bonds which stabilize the shape of the molecule. The LD complexes can be represented as helices in which the same interactions are responsible for the stability of the molecule. These helices can also be formed by opening the $\mathrm{CD}$ ring and connecting the rings to each other. Figure 1 shows the assumed similarity in structure of both derivatives. Both dextrins can also be prepared without a complexing agent e.g. by spray drying. CD retains its crystallinity on this manipulation due to the head-tail binding of the ring, whereas LD loses its regular structure during storage. Therefore the empty helix is called 'meta-stable'.

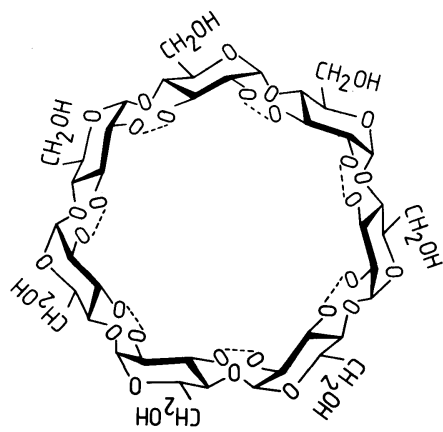

1

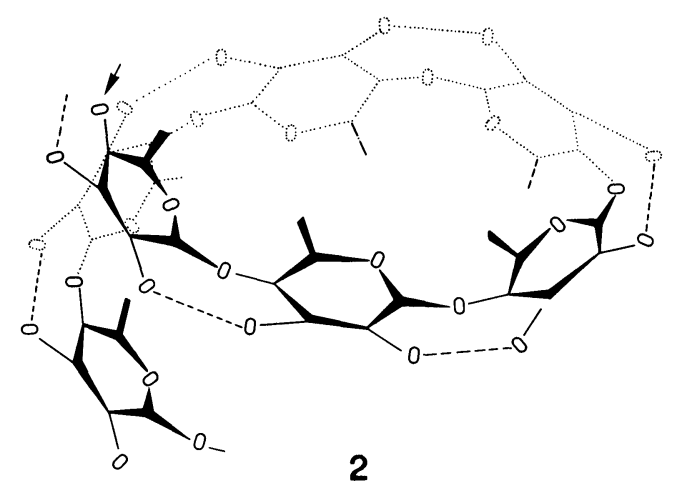

2

Figure 1 Steric structures of $\beta$-cyclodextrin (1) and linear dextrin complex (2) (only one winding is drawn here) 


\section{EXPERIMENTAL}

The starch derivatives were prepared and delivered by the Department of Biotechnology of TNO after drying and storage in dry Argon. All measurements were carried out on a Bruker 113v FTIR, operating at $2 \mathrm{~cm}^{-1}$ resolution using a TGS-detector for transmission spectroscopy and a MCT detector for Diffuse Reflectance Spectroscopy (DRIFT). The number of scans was 64 for transmission spectroscopy and 250 for DRIFT measurements. The apodization function was Happ Genzel and the zero filling factor was $2 . \mathrm{KBr}$ was used as matrix diluter in all measurements.

All experiments were carried out in two- or threefold. Resolution enhancement was attained by Fourier self-deconvolution for all resulting spectra using standard Bruker software. The assumed lineshape was Lorentzian with a half band width of $20 \mathrm{~cm}^{-1}$. The resolution enhancement factor chosen was 1.5.

\section{RESULTS AND DISCUSSION}

Figure 2 shows the DRIFT spectra after Fourier self-deconvolution of $\beta$-CD and LD n-butanol complexes (butanol content: $5 \%$ ) and meta-stable LD. The complex absorption pattern of $\beta$-CD between 1250 and $900 \mathrm{~cm}^{-1}$ appears to consist of eight individual bands, with maxima at 1156,1125, 1104, 1080, 1057, 1026, 999 and $944 \mathrm{~cm}^{-1}$. The spectrum of meta-stable LD shows an additional maximum at $1040 \mathrm{~cm}^{-1}$. Although these changes in spectra of retrograded starch were reported earlier (1), a full explanation has not yet been found. Detailed comparison of the three spectra shows an increasing line sharpening on comparing $\beta$-CD to the LD complex and finally the meta-stable LD. Therefore a simple relationship between crystallinity and line sharpening is proposed: increased order of the configuration of the derivative correlates with more pronounced absorption bands in this IR region.

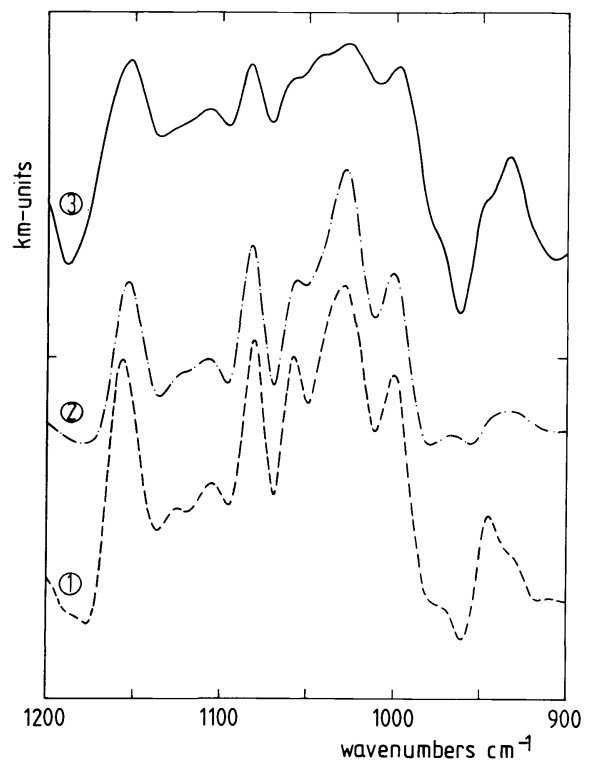

Figure 2 Deconvoluted DRIFT spectra of $\beta$-cyclodextrin and linear dextrin complex (2) and meta-stable linear dextrin (3)

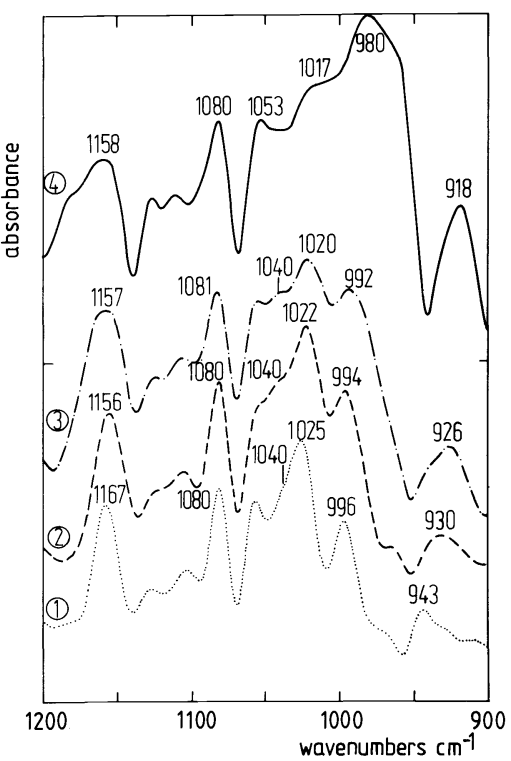

Figure 3 Deconvoluted absorption spectra of $\beta$-cyclodextrin (1), linear dextrin complex (2), meta-stable linear dextrin (3) and amorphous amylose (4)

Figure 3 shows the absorption spectra of the three samples and additionally of amorphous amylose (Dp 1000). The absorption band at $1040 \mathrm{~cm}^{-1}$ in the spectrum of the meta-stable LD is more pronounced than in the DRIFT spectra. However, the increasing trend of this absorption band as well as broadening of all the bands in this region, on comparing $\beta-C D$ to the LD complex and finally the meta-stable derivative is the same as observed in the DRIFT spectra. Of course this tendency in line broadening is observed in the spectrum of amorphous amylose, but the absorption pattern near $1000 \mathrm{~cm}^{-1}$ also changed dramatically.

Studying the spectra in more detail shows shifts of absorption bands with increasing or decreasing intensities (for the maxima near $1055,1020,990$ and $930 \mathrm{~cm}^{-1}$ ). These shifts correlate with the crystallinity of the compounds. In more detail the maxima 
are displayed in Figure3. To understand the observations described above, we refer to the assumed conformations of the starch derivatives. The line broadening is related to the variation in orientation of the functional groups within the molecule. The more crystalline samples have less possible conformations, and hence smaller distributions of bond energies, resulting in sharp lines in the spectra. Line broadening in this case is not caused by increasing water content, as has been shown earlier $(3,4)$. The band shifts observed and the decreasing or increasing intensities near $1000 \mathrm{~cm}^{-1}$ are more difficult to understand, but can be described to changes in the molecular environment of the $\mathrm{C}-\mathrm{OH}$ group at carbon 6 and the $\mathrm{C}-\mathrm{O}-\mathrm{C}$ linkages in the glucopyranose ring and the -D-glucosidic bindings. The primary alcohol group in crystalline fractions is less intra- and intermolecular bridged than in amorphous fractions. In this specific case $\beta-C D$ and LD complexes are represented as in Figure 1, while amorphous fractions can be represented as at random orientated polymers. Therefore the absorption maximum of the C-OH stretching vibration near $1020 \mathrm{~cm}^{-1}$ in spectra of amorphous compounds will shift to a lower frequency compared to crystalline fractions. On the other hand the conformations of the glucose polymers in crystalline fractions described here are characterized by a high degree of symmetry. It influences the degree of dipole moment variation during vibration of certain functional groups, which is observed as changes in intensities of the absorption bands.

Although these assumptions are plausible, further proof of the explanation given for the spectra of crystalline and amorphous starch derivatives is still necessary and can be obtained by comparing spectra of deuterated compounds with the undeuterated species; these experiments are carried out at this moment in our laboratory.

\section{CONCLUSIONS}

The conformation of starch derivatives can be studied successfully by infrared spectroscopy. The most interesting part of the spectrum is located between 1200 and $900 \mathrm{~cm}^{-1}$. In this region sharpening and shifting of the absorption bands, as well as changing of band intensities depend on the tertiary structure of the glucose polymers. Furthermore the assumed similarity in structure of $\beta-C D$ and LD complexes is confirmed by this FTIR study.

\section{REFERENCES}

1) R.H. Wilson, M.T. Kalichevsky, S.G. Ring and P.S. Belton, Carbohydr. Res. 166 (1987), 162-165.

2) Hayashibara Company, Okayama-shi, Japan, Patent No: 165500 NL. 1981.

3) R.H. Wilson and P.S. Belton, Carbohydr. Res., 180 (1988), 339-344.

4) S.F. Tanner, S.G. Ring, M.A. Whittam and P.S. Belton, Int. J. Biol. Macromol., 9 (1987), 219-224. 